\title{
AVALIAÇÃo DA BARREIRA HEMATO-ENCEFÁLICA NO TRANSPLANTE DE MEDULA ÓSSEA
}

\author{
SÉRGIO MONTEIRO DE ALMEIDA *, JOSÉ ANTONIO LVRAMENTO *, \\ RICARDO PASQÜINI **, VIVIANA BOCCARDI PALOU ***, \\ APARECIDO MENDES DE OLIVEIRA ${ }^{* * * *}$, ELVIRA MISSAKO DOI ${ }^{* * * * *}$,

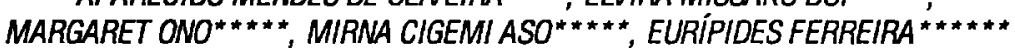

RESUMO - A barreira hemato-encefálica (BHE) contribui para o isolamento imunológico do sistema nervoso central (SNC). Sua avaliação nunca foi realizada em pacientes submetidos a transplante de medula óssea (TMO). Neste estudo a integridade da BHE foi avaliada através das proteínas do LCR, de forma quantitativa, a fim de observar a incidência e entender a fisiopatologia da doença do enxerto contra o hospedeiro crônica (DECH-C) no SNC. Foram estudadas amostras pareadas de LCR e soro de 33 pacientes com leucemia mielóide crônica submetidos a TMO alogênico, de doador aparentado, HLA idêntico. As amostras foram coletadas nos períodos pré TMO, pós TMO e concomitante à DECH-C. Nāo foi evidenciada quebra de BHE durante a DECH-C em nenhum dos casos estudados.

PALAVRAS-CHAVE: doença do enxerto contra o hospedeiro, barreira hemato-encefálica, auto-imunidade, líquido cefalorraquidiano, transplante de medula óssea, albumina.

\section{Blood-brain barrier evaluation in bone marrow transplantation}

ABSTRACT - The blood-brain barrier (BBB) contributes to the central nervous system (CNS) immunological isolation. BBB has never been studied in patients who developed chronic graft-versus-host disease (GVHD) after allogeneic bone marrow transplants (BMT), from HLA identical related donors. BBB disruption was investigated through the cerebrospinal fluid (CSF) proteins, quantitative and graphically, in order to detect the incidence and possible pathophysiology of the CNS involvement in chronic GVHD. Thirty three CSF and matched serum samples from chronic myeloid leukemia patients were collected pre BMT, pos BMT and during chronic GVHD. There was no evidence of BBB disruption in any patient studied.

KEY WORDS: graft-versus-host disease, blood-brain barrier, auto immunity, cerebrospinal fluid, bone marrow transplantation, albumin.

O sistema nervoso central (SNC) é considerado um local imunologicamente privilegiado, que não participa nas reações imunes do resto do organismo². Isso porque no SNC elementos normalmente identificáveis do sistema imunológico são raros ou inexistentes ${ }^{4,11,15}$. Além disso a existência de uma barreira funcional, a barreira hemato-encefálica (BHE), impede a difusão de macromoléculas como imunoglobulinas, complemento e fatores de coagulação, e inibe o tráfego de

Estudo realizado na disciplina de Neurologia e Serviço de TMO do Hospital de Clínicas da Universidade Federal do Paraná (HC-UFPR), Disciplina de Imunologia da Escola Paulista de Medicina - Universidade Federal de São Paulo (EPM-UNIFESP) e Centro de Investigações em Neurologia da Faculdade de Medicina da Universidade de São Paulo (FMUSP): *Neurologista; **Professor Titular, Disciplina de Hematologia; ***Biologista; ****Biomédico; *****Bioquímica; ******Professor Adjunto, Disciplina de Imunologia. Projeto realizado com apoio da FAPESP (932705-6) e CAPES. Aceite: 20-agosto-1997. 
células do sistema imunológico" ${ }^{11}$ Porém este conceito de isolamento imunológico vem sendo questionado, mostrando haver envolvimento do SNC em reaçōes imunológicas sistêmicas importantes como a doença do enxerto contra o hospedeiro (DECH) ${ }^{9,12,14,20,21,33,36,39}$.

A DECH ocorre em 30 a $60 \%$ dos pacientes submetidos a transplante de medula óssea (TMO), e constitui-se na sua principal complicação e causa de morbidade e mortalidade ${ }^{3,6}$. $O$ fenômeno de DECH foi inicialmente descrito por Barnes \& Loutit (1955) em cobaias irradiadas. Apesar de se recuperarem da irradiação e aplasia de medula, estas morriam subseqüentemente de "doença secundária"2y. Posteriormente, Santos \& Cole encontraram os mesmos achados em cobaias infundidas com medula óssea alogênica ${ }^{30}$. Observou-se que aumentando o número de células linfóides infundidas, a severidade da $\mathrm{DECH}$ aumentava e o tempo entre o TMO e início da doença diminuía, confirmando um mecanismo de reação do enxerto contra o hospedeiro ${ }^{30}$. Em humanos, a primeira descrição de DECH foi feita por Mathé et al, no início da década de sessenta ${ }^{19}$. A DECH se apresenta como duas síndromes distintas, que diferem tanto no tempo de início como nas suas caracteristicas clínicas $e$ fisiopatológicas, DECH aguda (DECH-A) e DECH crônica (DECH-C) ${ }^{37}$. A DECH-A, fisiopatologicamente, representa uma reaçāo alogênica dos linfócitos $T$ contra os antígenos de histocompatibilidade do receptor, mais especificamente antígenos menores de histocompatibilidade. Trata-se, portanto, de uma doença aloreativa $a^{37}$.

A DECH-C, ponto de atenção deste estudo, tem início acima de 100 dias após o transplante, e apresenta manifestações clínicas semelhantes às doenças do colágeno ${ }^{26,31}$. Fisiopatologicamente, representa uma reação auto-imune, devida a linfócitos $T$ auto-reativos, que não foram adequadamente destruidos no timo ${ }^{37}$. Estudos experimentais sustentam fortemente esta origem auto-imune na DECH$\mathrm{C}^{25}$. Quanto à forma de início, pode ser classificada em: progressiva, quando a $\mathrm{DECH}-\mathrm{A}$ progride gradualmente para DECH-C; quiescente, quando há resolução da DECH-A e desenvolvimento posterior da DECH-C; de novo quando há início da DECH-C sem evidência clínica ou histológica de DECH-A anterior ${ }^{31}$. A Tabela 1 apresenta a classificaçāo clínico-patológica da DECH-C ${ }^{31}$. Ainda não está bem estabelecido se a DECH pode afetar diretamente o $\mathrm{SNC}^{23}$. Várias séries publicadas, tanto clínicas como de necropsias, não encontraram envolvimento do SNC diretamente relacionado com DECH ${ }^{27}$. Porém, alguns estudos experimentais ${ }^{11,14}$, e alguns relatos clínicos ${ }^{13,18,28,38}$, procuram mostrar este envolvimento, especialmente na DECH crônica.

Devido às semelhanças entre doenças auto-imunes e a DECH-C, existe a possibilidade de quebra da BHE, como ocorre em algumas colagenoses. Frente a essa possibilidade, este estudo objetiva avaliar como se comporta a integridade da BHE, em pacientes submetidos a TMO, em especial durante a DECH-C. Apesar de haver a possibilidade de alteração da BHE durante a DECHC, este aspecto da doença não foi ainda avaliado.

Tabela 1. Classificação clínico-patológica da DECH crônica" ${ }^{31}$.

\section{DECH CRÔNICA LIMITADA}

Um ou ambos

1. Envolvimento localizado da pele;

2. Disfunção hepática devido à DECH crônica.

\section{DECH CRÔNICA EXTENSA}

Um dos:

1. Envolvimento generalizado da pele; ou

2. Envolvimento localizado da pele e/ou disfunçăo hepática devido a DECH crônica, associada com:

a- Histologia hepática mostrando hepatite crônica agressiva, necrose em ponte,ou cirrose; ou

b- Envolvimento ocular (teste de Schirmer com menos de $5 \mathrm{~mm}$ ); ou

c- Envolvimento de glândula salivar menor ou mucosa oral demonstrado em biópsia de lábio; ou

d- Envolvimento de qualquer outro órgão alvo. 


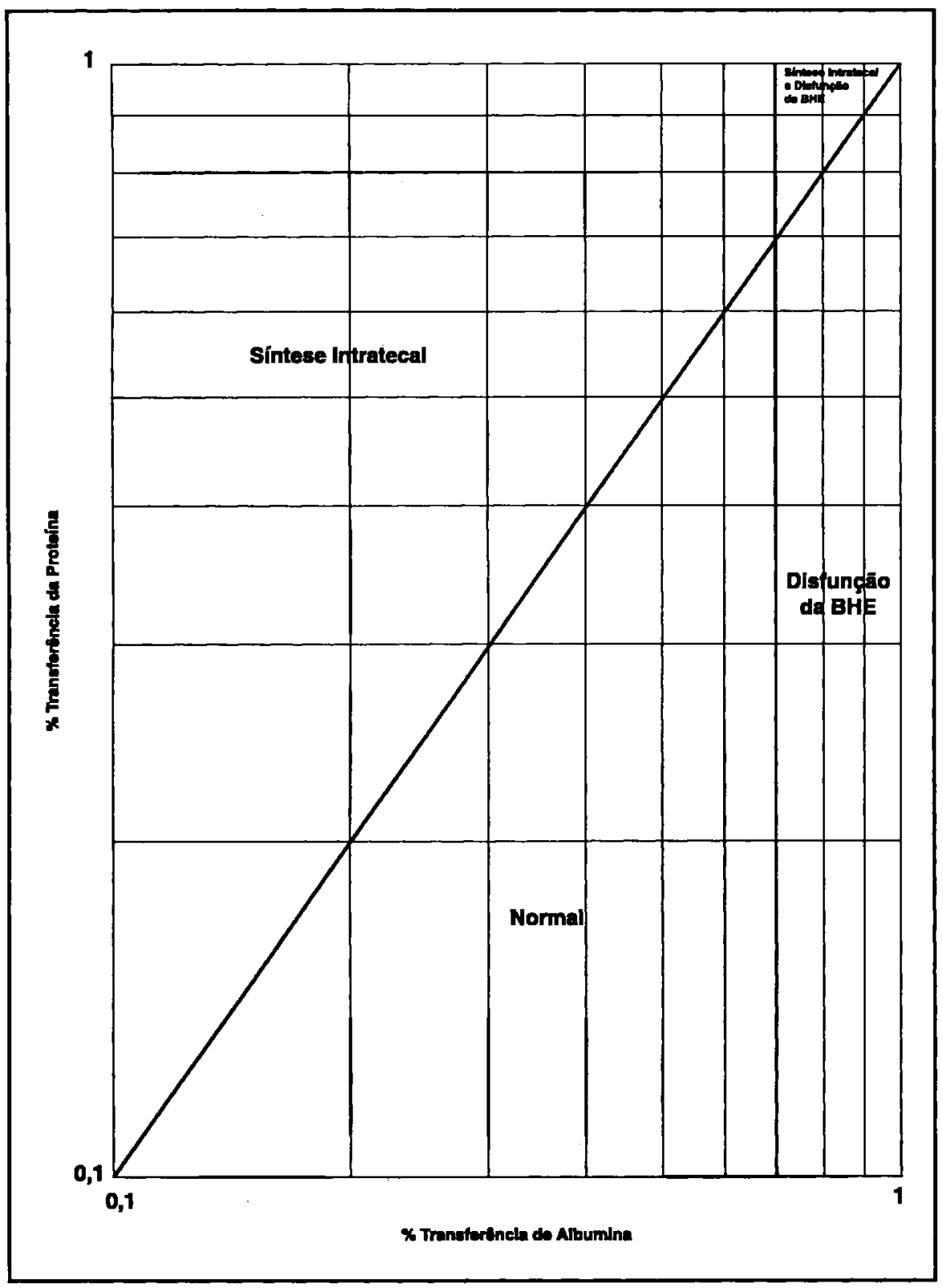

Fig 1. Avaliação da BHE (Thompson"', 1988).

\section{CASUÍSTICA E MÉTODOS}

A casuística e os métodos utilizados para quantificação dos valores proteicos foram descritos no artigo anterior'.

A integridade da BHE foi avaliada através de: proteína total no LCR $^{3 \text {; }}$ valor absoluto de albumina no LCR5; quociente de albumina, Alb LCR / Alb soro ${ }^{35}$. 
Tabela 2. Avaliação da integridade da BHE, porcentagem de casos alterador, no grupo controle e nos períodos Pré, Pós e DECH-C.

\begin{tabular}{|c|c|c|c|c|c|c|c|c|}
\hline & \multicolumn{2}{|c|}{ Controle $n=30$} & \multicolumn{2}{|c|}{ Pré $n=33$} & \multicolumn{2}{|c|}{ Pós $n=27$} & \multicolumn{2}{|c|}{ Dech-c $n=17$} \\
\hline & alt. & $\%$ & alt. & $\%$ & alt. & $\%$ & alt. & $\%$ \\
\hline $\mathrm{PT}(\mathrm{lcr})$ & 0 & 0 & 5 & 15,15 & 4 & 14,81 & 3 & 17,65 \\
\hline Alb. (lcr) & 0 & 0 & 1 & 3,03 & 1 & 3,70 & 0 & 0 \\
\hline Q Alb. & 0 & 0 & 1 & 3,03 & 1 & 3,70 & 0 & 0 \\
\hline
\end{tabular}

Graficamente a integridade da BHE foi avaliada relacionando-se o quociente de cada proteína (relação soro/LCR) com o respectivo tamanho de sua molécula em Angströns $(\AA)$. Para a albumina o raio considerado foi $35,8 \AA$, para a2 macroglobulina foi $93,5 \AA$, para $\operatorname{IgG} 53 \AA$, IgA $57 \AA$, IgM $121 \AA^{7.34}$. Os valores inferiores normais dos quocientes de albumina e a2 macroglobulina foram considerados, respectivamente, 150 e $500^{7}$. A graduaçāo dos distúrbios de BHE foram determinados graficamente e classificados de acordo com os quocientes de albumina (soro/LCR) em leve, 70-130; moderado, 30-70; grave, 10-30 e quebra de BHE quando abaixo de $10^{32}$. A integridade da BHE foi avaliada, ainda, pelo gráfico $\log / \log (\text { Fig } 1)^{34}$.

\section{RESULTADOS}

Os casos com alteração da integridade da BHE estão descritos na Tabela 2.

No grupo controle, graficamente nenhum caso apresentou quebra de BHE. Houve quebra da BHE, classificadas como leve, em um caso no pré e em outro no pós TMO. Durante o período de DECH-C não foi encontrada quebra de BHE, em nenhum caso, por nenhum dos métodos estudados (Gráfico 1).

Analisadas no conjunto, as médias de todas as variáveis para investigação da integridade da BHE se encontram dentro dos limites normais (Tabela 3).

\section{DISCUSSĀO}

O conceito de uma barreira que limita as trocas entre o sangue e o encéfalo foi iniciado pelas observaçōes de Ehrlich (1885) ${ }^{24}$. O capilar cerebral é a estrutura anatômica responsável pela BHE e as "tight junctions" (zonulae occludens), existentes nas células endoteliais, servem como bases morfológicas. Além disso, fendas intracelulares, vesículas de pinocitose e fenestras, que permitem a troca transcapilar na maioria dos capilares sistêmicos, não são encontradas nas células endoteliais do SNC. As células endoteliais dos capilares do SNC possuem, ainda, uma membrana de base proeminente e são ricas em mitocôndrias que são raras nas células endoteliais sistêmicas. Os capilares cerebrais são circundados pelos processos dos astrócitos tipo 1, chamados de "pés astrocitários". Estas células gliais especializadas induzem as células endoteliais a formar as "tight junctions". Esta estrutura anatômica possui a complexa função de excluir a transferência de moléculas grandes da circulação, embora permanecendo permeável para água e lipídios. A taxa de transferência pela BHE está relacionada com o peso molecular, o raio da molécula, assim como com sua carga elétrica ${ }^{4 \times .24}$.

Neste estudo foram incluídos apenas pacientes com LMC, pelo fato de não serem submetidos a quimioterapia intratecal e/ou radioterapia, para profilaxia do envolvimento da doença de base no SNC, que poderiam levar à alteração na BHE. O envolvimento do SNC na LMC é extremamente raro. Nenhum paciente incluído neste estudo apresentou alteração neurológica, nos periodos em que o LCR foi coletado.

Nos casos da literatura, que procuram provar um envolvimento direto do SNC pela DECH-C, apenas dois possuem estudo de LCR. Estes casos apresentavam sintomatologia neurológica e as alterações no SNC investigadas por imuno-histoquimica. O LCR estava normal em um caso ${ }^{18} \mathrm{e}$ no outro havia alteração de celularidade e aumento moderado de proteínas ${ }^{13}$. Em ambos não foi realizado um estudo proteico mais detalhado, o que impede maiores ilaçōes quanto à integridade da BHE. 


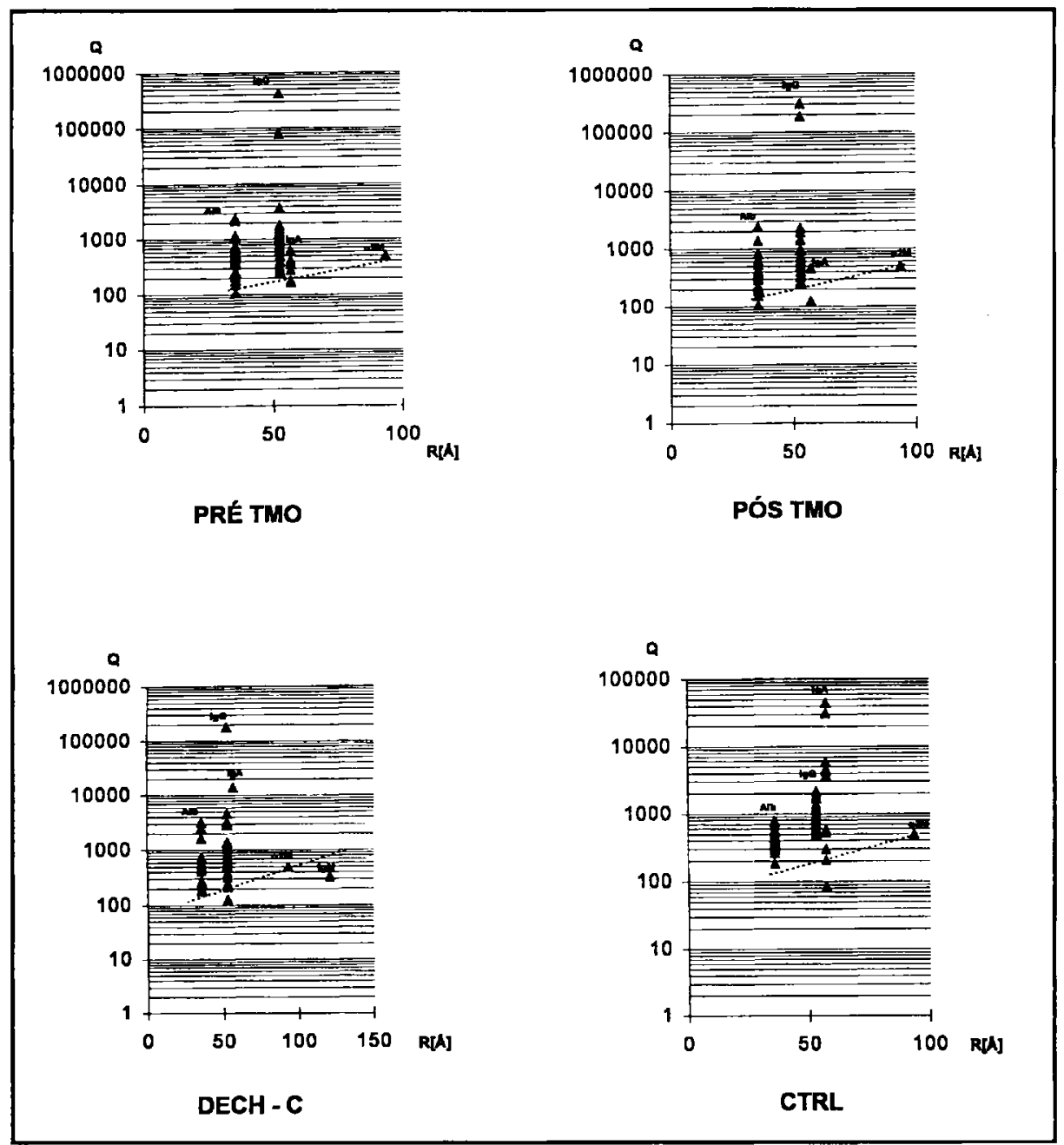

Gráfico l. Comportamento das moléculas protêicas, frente a $B H E$, nas várias fases do TMO. A linha tracejada indica o limite inferior da BHE normal. Ordenada: quociente soro/LCR. Abscissa: raio da molécula (A). Albumina (Alb), $\infty 2$ macroglobulina ( $\propto 2 M)$.

Tabela 3. Avaliação da integridade da BHE, no grupo controle e nos periodos Pré, Pós e DECH-C.

\begin{tabular}{|c|c|c|c|c|c|c|c|c|c|c|c|c|}
\hline & \multicolumn{3}{|c|}{ Controle $n=30$} & \multicolumn{3}{|c|}{ Pré $n=33$} & \multicolumn{3}{|c|}{ Pós $n=27$} & \multicolumn{3}{|c|}{ Dech-c $n=17$} \\
\hline & $\overline{\mathbf{X}}$ & DP & Md & $\overline{\mathbf{X}}$ & DP & Md & $\overline{\mathbf{X}}$ & DP & Md & $\overline{\mathbf{X}}$ & DP & Md \\
\hline PT (lcr) & 23,50 & 8,03 & 23,00 & 32,91 & 12,97 & 31,00 & 32,17 & 12,49 & 28,00 & 34,70 & 17,96 & 31,00 \\
\hline Alb. (lcr) & 15,61 & 4,17 & 15,95 & 15,63 & 10,04 & 12,92 & 17,27 & 9,99 & 15,17 & 14,40 & 8,64 & 15,98 \\
\hline Q Alb. & 0,002 & 0,0008 & 0,002 & 0,003 & 0,002 & 0,002 & 0,003 & 0,002 & 0,003 & 0,003 & 0,002 & 0,002 \\
\hline
\end{tabular}


Para a avaliação da integridade da BHE são utilizadas como marcadores proteínas com peso molecular de intermediário a clevado, o que impede uma passagem espontânea, e que são produzidas exclusivamente fora do SNC, como a albumina, $\alpha 2$ macroglobulina e a transferrina. Além disso estas proteínas apresentam dosagem sérica relativamente constante ${ }^{34}$.

Neste estudo a variável que apresentou maior número de casos alterados, em todas as fases do TMO, foi a proteína total no LCR, porém para avaliação da integridade da BHE este é o índice de menor sensibilidade e especificidade. Tanto graficamente como pelos índices calculados, não foi encontrado, nesta casuística, alteração de BHE durante o período de DECH-C e apenas em casos isolados nas outras fases do TMO. Houve concordância entre os vários métodos utilizados na avaliação.

Os resultados deste estudo corroboram o trabalho de Kajiwara et al. ${ }^{14}$, realizado em animais de experimentação, nos quais foi induzido $\mathrm{DECH}$, a $\mathrm{BHE}$ sendo investigada através da infusão de corante azul de Evans. Em todos os casos a BHE apresentou-se íntegra.

Pode-se concluir que não há quebra de BHE devido a DECH na forma crônica. O envolvimento direto desta no SNC, se ocorre, se faz por outros mecanismos, provavelmente através de alteração na expressividade dos antígenos de histocompatibilidade em associação com linfócitos auto-reativos, independente da existência de quebra de BHE.

Agradecimento - Ao Prof. Dr. Lineu César Werneck.

\section{REFERÊNCIAS}

1. Almeida SM, Livramento JA, Pasqüini R, Palou VB, Oliveira AM, Doi EM, Ono M, Aso MC, Ferreira E. Avaliaçāo da sintese intratecal de imunoglobulinas no transplante de medula óssea. Arq Neuropsiquiatr 1997;55:801-811.

2. Barker CF, Billingham RE. Immunologically privileged sites. Adv Immunol 1977;25:1-53.

3. Barrett J. Graft versus host disease. In:Treleaven $J$, Barrett $J$ (eds). Bone marrow transplantation in practice. Edinburgh:Churchill Livingstone, 1992:257-272.

4. Bradbury MW. The concept of a blood brain barrier. London: John Wiley \& Sons, 1979.

5. Breebart L, Becker H, Jongebloed FA. Investigation of reference values of components of CSF. J Clin Chem Clin Biochem 1978;16:561-565.

6. Deeg HJ, Henslee-Downey PJ. Management of acute graft-versus-host disease. Bone Marrow Transpl 1990;6:1-8.

7. Felgenhauer K, Schliep G, Rapic N. Evaluation of the blood-CSF barrier by protein gradients and the humoral immune response within the central nervous system. I Neurol Sci 1976:30:113-128.

8. Fishman RA. Cerebrospinal fluid in diseases of the nervous system. Ed2. London: W.B. Saunders, 1992.

9. Hauser SL, Bhan AK, Gilles FH, Hoban CJ, Reinherz EL, Schlossman SF, Weiner HL. Immunohistochemical of humam brain with monoclonal antibodies that identify lynphocytes, monocytes la antigens. J Neuroimmunol 1983;5:197-208.

10. Henry JB. Clinical and diagnosis management by laboratory methods. Ed18. Philadelphia: WB Saunders, 1991.

11. Hickey WF. Microglial participation in the initiation of CNS inflammation. Disc Neurosc 1993;9:74-78.

12. Hickey WF, Kimura H. Graft vs. host disease elicits expression of class I and class II histocompatibility antigens and the presence of scattered T lymphocytes in rat central nervous system. Proc Natl Acad Sci USA 1987;84:2082-2086.

13. Iwasaki Y, Sako K, Ohara Y, Miyazawa M, Minegishi M, Tsuchiya S, Konno T. Subacute pan encephalitis associated with chronic graft-versus-host disease. Acta Neuropathol 1993;85:566-572.

14. Kajiwara K, Hirozane A, Fukumoto T, Orita T, Nishizaki T, Kamiryo T, Ito H. Major histocompatibility complex expression in brain of rats with graft-versus-host disease. J Neuroimmunol 1991;32:191-198.

15. Lampson LA, Hickey WF. Monoclonal antibody analysis of MHC expression in human brain biopsies:tissue ranging from histologically normal to that showing different levels of glial involvement. J Immunol 1986;136:4054-4062.

16. Link $\mathrm{H}$, Tibbling $\mathrm{G}$. Principles of albumin and $\mathrm{IgG}$ analyses in neurological disorders. II.Relation of the concentration of the proteins in serum and cerebrospinal fluid. Scand J Clin Lab Invest 1977;37:391-396.

17. Mancini G, Carbonara AO, Heremans JF. Immunochemical quantitation of antigens by single radial immunodiffusion. Immunochemistry 1965;2:235-254.

18. Marosi C, Budka H, Grimm G, Zeithofer J, Sluga E, Brunner C, Schneeweiss B, Volc B, Bettelheim P, Panzer S, Kier P, Graninger W. Haas $O A$, Hinterberger W. Fatal encephalitis in a patient with chronic graft-versus-host disease. Bone Marrow Transpl 1990;6:53-57.

19. Mathe G, Amiel JL, Schwarzenberg L, Cattan A, Schneider M. Haematopoietic chimera in man after allogenic (homologous) bone-marrow transplantation:control of the secondary syndrome. Specific tolerance due to the chimerism. Br Med J 1963;28:1633-1635.

20. Matsumoto $Y$, Fujiwara $M$. In situ detection of class 1 and class II major histocompatibility antigens in the rat central nervous system during experimental allergic encephalomyelitis: an immunohistochemical study. J Neroimmunol 1986:12:265-277.

21. Matsumoto Y, Hara N, Tanaka R, Fujiwara M. Immunohistochemical analysis of the rat central nervous system during experimental allergic encephalomyelitis, with special reference to Ia-positive cells with dentritic morphology. I Immunol 1986;136:3668-3676.

22. Meulemans O. Determination of total protein in spinal fluid with sulphosalicylic acid and trichloro-acetic acid. Clin Chim Acta $1960 ; 5: 757-761$. 
23. Openshaw $H$, Slatkin NE. Neurological complications of bone marrow transplantation. In:Forman SJ, Blume KG, Thomas ED (eds). Bone marrow transplantation. Boston: Blackwell, 1994:.482-496.

24. Pardridge WM. The blood-brain barrier:cellular and molecular biology. New York:Raven Press, 1993.

25. Parkman R. Clonal analysis of murine graft-vs-host disease:I.Phenotypic and functional analysis of $T$ lymphocyte clones. J Immunol 1986;136:3543-3547.

26. Parkman R. Graft-versus-host disease and cytokines. Cur Op Hematol 1993:223-237.

27. Patchell RA, White III CL, Clark AW, Beschorner WE, Santos GW. Neurologic complications of bone marrow transplantation. Neurology 1985;35:300-306.

28. Rouah E, Gruber R, Shearer W, Armstrong D, Hawkins EP. Graft-versus-host disease in central nervous systern:a real entity? Am J Clin Pathol 1988;89:543-546.

29. Santos GW. History of bone marrow transplantation. Clin Haematol 1983;12:611-639.

30. Santos GW, Cole L. Effects of donor and host lymphoid and myeloid tissue injections in lethally X-irtadiated mice treated with rat bone marrow, J Nat Cancer Inst 1958;21:279-293.

31. Shulman HM, Sullivan KM, Weiden PL, McDonald GB, Striker GE, Sale GE, Hackman R, Tsoi M, Storb R, Thomas ED. Chronic graft-versus-host syndrome in man: a long-term clinicopathologic study of 20 Seattle patients. Am J Med 1980;69:204217.

32. Schliep G, Felgenhauer K. Serum-CSF protein gradients, the blood-CSF barrier and local immune response. J Neurol 1978;218:77-96.

33. Sobel RA, Ames MB. Major histocompatibility complex molecule expression in the human central nervous system:immunohistochemical analysis of 40 patients. J Neuropathol Exp Neurol 1988;47:19-28.

34. Thompson, EJ. The CSF Proteins:a biochemical approach. Amsterdam:Elsevier, 1988.

35. Tibbling G, Link H, Ohman S. Principles of albumin and IgG analyses in neurological disorders. I. Establishment of reference values. Scand J Clin Lab Invest 1977;37:385-390.

36. Traugott U, Scheinberg LC, Raine CS. On the presence of Ia-positive endothelial cells and astrocytes in multiple sclerosis lesions and its revelance to antigen presentation. J Neuroimmunol 1985;8:1-14.

37. Van Bekkum DW. What is graft versus host disease? Bone Marrow Transpl 1991;7(Suppl 2):110-111.

38. Wiznitzer M, Packer RJ, August CS, Burkey ED. Neurological complications of bone marrow transplantation in childhood. Ann Neurol 1984;16:569-576.

39. Woodroofe MN, Bellamy AS, Feldman M, Davison AN, Cuzner ML. Immunocytochemical characterisation of imune reaction in the central nervous system in multiple sclerosis:possible role for microglia in lesion growth. J Neurol Sci 1986;74:135-152. 